\title{
Prostate Cancer Survivorship: A Systematic Review
}

\section{Sanchia S Goonewardene ${ }^{1 *}$, Persad $R^{2}$, Nanton $V^{3}$, Young $A^{3}$ and Makar $A^{4}$}

${ }^{1}$ Guys Hospital, Kings College London, UK

${ }^{2}$ Bristol Southmead, UK

${ }^{3}$ University of Warwick, UK

${ }^{4}$ Worcestershire Acute Hospitals, Worcestershire, UK

\begin{abstract}
Background: Due to advances in cancer diagnosis and treatment, the number of prostate cancer survivors are increasing. Yet, with this expanding cohort of patients, very little has been done to develop services.

Objective: A systematic review was conducted to explore prostate cancer survivorship issues. This analysis will inform development of interventions.

Design/setting: A systematic review was conducted using the following databases from 2000 to Decembers 2013: CINAHL and MEDLINE (NHS Evidence), Cochrane, AMed, BNI, EMBASE, Health Business Elite, HMIC, PschINFO. The papers were retrieved and a quality assessment was conducted using a new tool for survivorship care standards.

Participants/Interventions/ Outcome measurements/ results: 76 papers met the criteria for inclusion. These specified papers must be on primary research, related to prostate cancer AND Survivorship OR any one of the categories of nutrition, exercise therapy, psychology, treatment outcomes.

Discussion: The literature is reviewed and the way forward for survivorship discussed. We also identify possible themes for research.

Patient summary: Based on these results, we develop a prostate cancer survivorship care assessment tool and identify areas of practice that can be targeting for further research.
\end{abstract}

Keywords: Prostate cancer; Survivorship; Patient care; Community based follow-up

\section{Introduction}

Over 2 million people in England have a diagnosis of cancer (National Cancer Survivorship Initiative, 2008). Of this, over 250,000 have been diagnosed with prostate cancer [1]. And 130,000 people per year die [2]. The Department of Health is spending $£ 750$ million on improving earlier diagnosis and prevention of cancer. During the next decade, a rapid increase in the number of new cancer diagnoses as well as a growing number of cancer survivors are predicted [3].

Hospital clinics are often overbooked with follow-up patients, with little time available for each patient. Yet few studies or guidelines address the broader, multifaceted aspects of cancer survivorship including self-responsibility and patient empowerment [4].

The Quality, Innovation, Productivity and Prevention (QIPP) transformational programme has been set up by the Department of Health to improve the quality of care the NHS delivers while making up to $£ 20$ billion of efficiency savings by 2014-15. One component involves risk profiling of patients, supported by community based teams and developing shared care/ decision making. As a result any programme which is set up must do the same. This reflects on the cancer survivorship programme, as the basis of this programme, is risk stratification, according to likelihood of recurrence.

\section{Cancer Survivorship}

A cancer survivor is any person who has received a diagnosis of cancer from diagnosis until the end of life. Survivorship is defined by Macmillan Cancer Support, a leading UK cancer care and support charity, as someone who has completed initial cancer management with no evidence of apparent disease. According to the National Cancer Institute, cancer survivorship encompasses the "physical, psychosocial, and economic issues of cancer from diagnosis until the end of life." (National Cancer Institute).

Prostate cancer survivors require further investigation as there are concerns current follow-up methods are unsuitable [5]. Due to the growing population of survivors of prostate cancer and the period of austerity for the NHS, patients are not getting the holistic care required during the survivorship phase. Concerns regarding permanent physical, psychosocial, and economic effects of cancer treatment were highlighted by the US Institute of Medicine Report [6]. This defined landmarks for survivorship care: monitoring for recurrence, metastases or side effects and coordination between secondary and primary care.

The unmet needs of cancer survivors, the rising numbers, and pressures to utilise resources efficiently [7] are a significant burden on the health system. These issues have been raised by the National Cancer Survivorship Initiative (NCSI) [8] which highlighted key shifts in attitude towards care.

The current method of follow-up involved focusing on cancer as an acute disease, with monitoring for recurrence, and no focus on the physical, social, emotional or psychological impact of being a Cancer Survivor. However, there is some debate as to the efficacy of this [7].

${ }^{*}$ Corresponding author: Sanchia S Goonewardene, MBChB BmedSc (Hons) PGCGC Dip, SSC MRCS (Ed) MRCS (Eng) Guys Hospital, Kings College London, UK, Tel: 00447717713036; E-mail: sanchi7727@gmail.com

Received October 23, 2014; Accepted May 03, 2015; Published May 27, 2015

Citation: Goonewardene SS, Persad R, Nanton V, Young A, Makar A (2015) Prostate Cancer Survivorship: A Systematic Review. J Health Edu Res Dev 3: 128 doi:10.4172/jherd.1000128

Copyright: (c) 2015 Goonewardene SS, et al. This is an open-access article distributed under the terms of the Creative Commons Attribution License, which permits unrestricted use, distribution, and reproduction in any medium, provided the original author and source are credited. 
Prostate Cancer recurrence can be followed up via PSA, without the actual need to come to clinic. Together with an older population, we have to start formulating pathways to get around this issue in an increasingly financially stricken NHS.

\section{Current Systematic Reviews}

Current systematic reviews on prostate cancer survivorship cover a range of topics.

These include symptoms include physical limitations, cognitive limitations, depression/anxiety, sleep problems, fatigue, pain, and sexual dysfunction [9]. This demonstrated cancer survivors can experience symptoms for more than 10 years following treatment. This also highlighted a need for evaluated and managed to optimize longterm outcomes. Another review has highlighted patient requirement for an active part in their healthcare during the survivorship phase [10]. The challenge is in integrating lifestyle support into standardised models of aftercare. EAU guidelines [11] do highlight the need for PSA follow-up in this cohort of patients.

Exercise was found to produce many beneficial effects in the cancer population including improvements in physical function, quality of life, body weight, fatigue levels, and psychological. This improved quality of life; decreased levels of anxiety, fatigue, and depression; and increased levels of functional capacity. Another review indicates exercise interventions are safe, resulting in improvements in physical fitness, QoL, fatigue, and psychosocial outcomes. Positive effects of exercise interventions are more pronounced with moderate- or vigorous exercise. Physical activity guidelines for cancer survivors suggest that physical activity should be an integral and continuous part of care for all cancer survivors [12]. This highlighted future studies should focus on identifying clinical, personal, physical, psychosocial, and intervention. More insight into the working mechanisms of exercise interventions on health outcomes in cancer survivors is needed to improve the efficacy and efficiency of interventions. The challenge, therefore, is in integrating lifestyle support into standardised models of aftercare for cancer survivors [10]. In addition, there is one Cochrane review on this topic, highlighting the importance of interventions to promote exercise including programme goals, prompting practise and self-monitoring and encouraging participants to attempt to generalise behaviours learned in supervised exercise environments. In this case, exercise prescriptions should be designed around individuals.

Diet and wellbeing is also important region for intervention in the survivorship cohort [13]. Exercise and diet interventions can be used to improve health and wellbeing of cancer survivors to develop maximally effective interventions as specified in that review.

Reviews also examined communication between families. Couples, regardless of gender, who are survivors of prostate cancer face a number of challenges and opportunities that impact their health, QOL, communication, and overall relationship satisfaction [14]. In addition, reviews have also highlighted self-management as a method of providing health-care solutions to ameliorate men's functional and emotional problems [15]. However, at the same time satisfied patients, patients with fulfilled information needs, and patients who experience less information barriers, in general have a better HRQoL and lower levels of depression and anxiety [16]. Other reviews have highlighted quality of life tools are lacking [17]. The role of the Nurse practitioner/ specialist nurse also has a strong impact on cancer survivorship care by serving in various roles and settings throughout the cancer trajectory to improve patient outcomes [18]. Reviews have also been conducted into prostate cancer support-groups: pen-ended, psychoeducational groups with large meetings, expert speakers, and structured, efficient organizations appear most beneficial [19].

\section{Method}

A systematic review was conducted. The search strategy (Figure 1) aimed to identify all references related to prostate cancer, survivorship, specific categories and treatment outcomes. (Prostate cancer or prostate neoplasms) and (survivorship or survivor) or (support care or diet therapy or exercise or communication) and (post therapy OR post treatment). Our selection criteria specified papers must be related to Prostate Cancer and Survivorship. The following databases were screened from 1984 to December 2013: CINAHL and MEDLINE (NHS Evidence), Cochrane, AMed, BNI, EMBASE, Health Business Elite, HMIC, PschINFO. In addition, searches using Medical Subject Headings (MeSH) and keywords were conducted using Cochrane databases. Primary research only was included in the the systematic review. Two UK-based experts were consulted in Survivorship care to identify additional studies.

\section{Eligibility}

Studies were eligible for inclusion if they reported primary research focusing on prostate cancer survivorship related to nutrition, psychology, physical therapy, treatment outcomes and communication and treatment outcomes. Papers were included if published after 1984 and had to be in English. Studies that did not conform with this were excluded (Figure 1).

\section{Selection criteria}

Abstracts were independently screened for eligibility by two reviewers and disagreements resolved through discussion or third opinion. Agreement level was calculated using Cohen's Kappa to test the intercoder reliability of this screening process. The PRISMA flow diagram demonstrates the results of the screening and selection process [ref]. According to criteria 76 papers were identified.

\section{Data Extraction and Quality Assessment of Studies}

Data extraction was piloted by SSG and amended in consultation

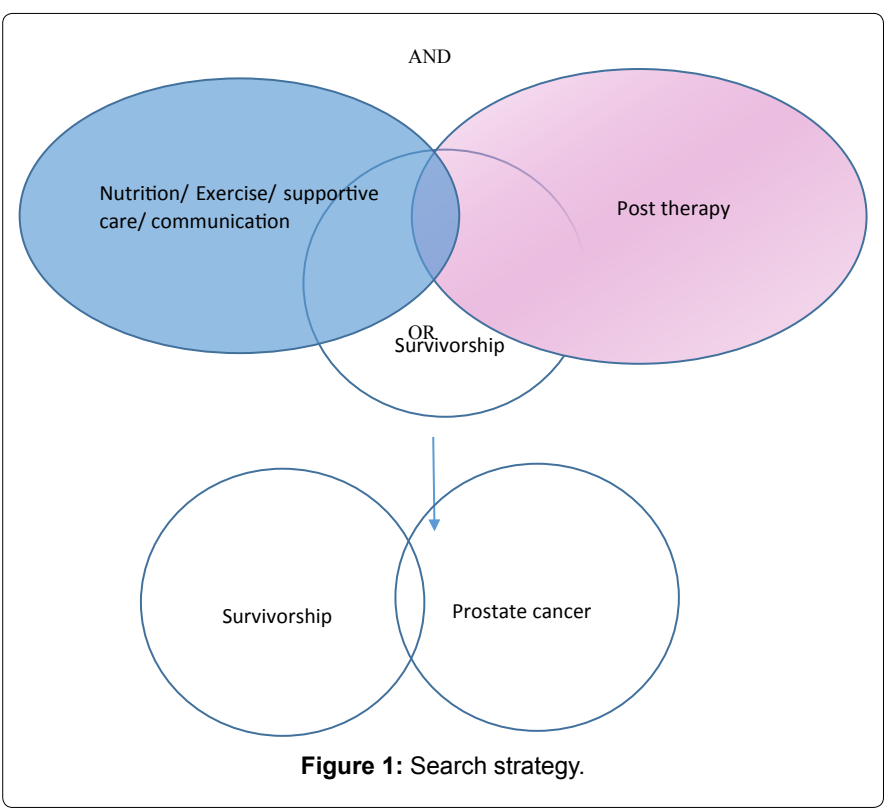


with the research team. Data extraction included authors, year and country of publication, study aims, setting, intervention aims, number of participants, study methods, intervention components and delivery methods, comparison groups and outcome measures, notes and followup questions for the authors. Included studies were quality assessed using for experimental studies, (Popay). for the action research and qualitative studies and the Critical Appraisal Skills Programme for retrospective studies. Individual quality assessment tools enabled us to focus on the specific study designs appropriately.

\section{Results}

Flow chart (adapted from Moher PRISMA) of studies related to Prostate cancer survivorship, holistic care, quality of life and followup is shown in Figure 2. The searches identified 2495 papers (Figure 2). However, only 76 mapped to search terms. 599 were excluded due to not being applicable to the topic. 1761 were duplicates. Of the 76 papers left, relevant abstracts were identified and the full paper obtained, all of which were in English. There was considerable heterogeneity (numbers/ method, expand) among the included studies therefore a narrative synthesis of the evidence was undertaken. Studies demonstrated a number of problems associated with prostate cancer survivorship care, they did not propose solutions to resolve the issues. What was also demonstrated was significant fracturing of prostate cancer survivorship care, which was cost inefficient and not properly addressing survivors' needs (Table 1).

\section{UK Studies}

Out of 76 papers, there were only 9 UK studies, indicating how far behind in prostate cancer survivorship care we are. However, the ones that were present in this systematic review highlight a number of requirements within survivorship care.

Faithfull [15] examined 22 participants as part of a quasiexperimental: feasibility study with 7 weeks of group and individual sessions. Outcomes were analysed via questionnaire based on urinary symptoms were measured before the intervention and again after 4 months of follow-up through International Prostate Symptom Scores This was conducted by researchers, This pilot study provides data suggesting that a narrowly targeted, cognitive and behavioural self-management intervention can improve LUTS in men who have had radiotherapy treatment for prostate cancer (Moher 'B' quality). This emphasises the importance of contact with secondary care in the survivorship phase [18]. Conducted a descriptive controlled analysis with over 18000 survivors vs controls, examining analysis of consultation rates/ 6 years. Prostate cancer requires $3 \mathrm{x}$ more consultations than controls. (Moher ' $\mathrm{B}$ ' quality). This again emphasises the same point.

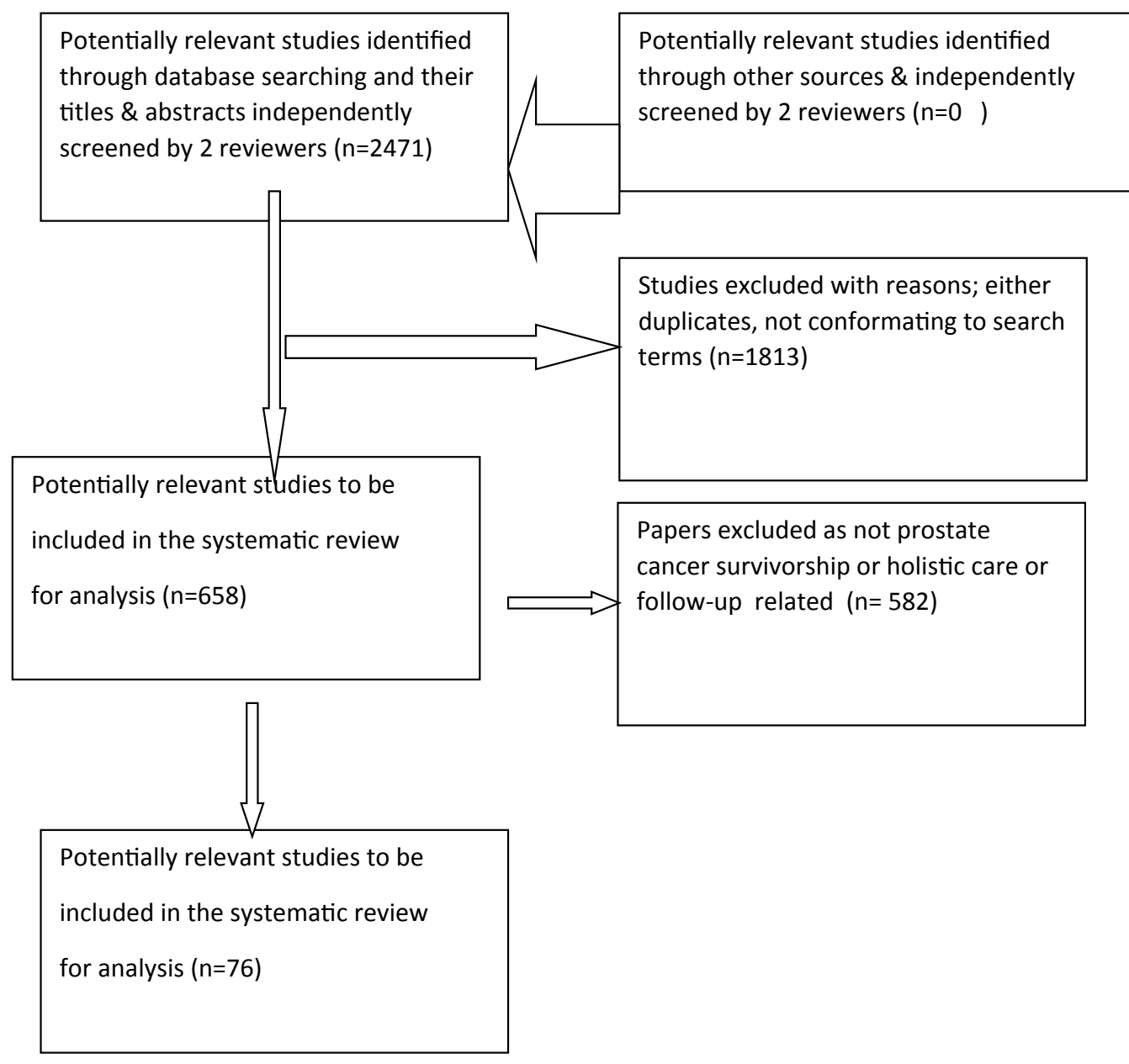

Figure 2: Results of PRISMA flow diagram for search strategy. 


\begin{tabular}{|c|l|}
\hline $\begin{array}{c}\text { Type of study design } \\
\text { Randomised controlled trials }\end{array}$ & Papers \\
\hline Randomised trial: & Perkins H., 2009 \\
\hline Cross-sectional studies: & $\begin{array}{l}\text { (Kent et al.) [32]. (Mols et al.) [16]. (Skolarus et al.) [39], (Del-Giudice et al.) [38]. (Miller et al.) [25]. (Schaefer et al.) [28]. (Taskilaa } \\
\text { et al.) [27]. (Lindbohn et al.) [27] }\end{array}$ \\
\hline Cross sectional case control & (Miller et al.) [25] \\
\hline Control- cross over study & (Demark-Wahnefried et al.) [36] \\
\hline Case- control study & (Mols et al.) [16]. (Synder et al.) [13]. (Skolarus et al.) [39]. \\
\hline Cohort Study & (Sheriff et al.) [37]. \\
\hline Quasi Experimental study & (Faithfull et al.) [15]. \\
\hline Experimental & Badger et al. [29]. \\
\hline Feasibility study & (Campbell et al.) [24], (Skolarus et al.) [39]. \\
\hline Descriptive study & (Skolarus et al.) [39], (Takisila et al.) [27]. \\
\hline Longitudinal study & (Taylor-Ford et al.) [31], (Skolarus et al.) [39] \\
\hline Qualitative studies & (Grunfield et al.) [39] (Faithfull et al.) [15] \\
\hline Descriptive controlled study & (Synder et al.) [34], (Del Giudice et al.) [38], (Grov) [30] \\
\hline
\end{tabular}

Table 1: Characteristics of studies.

\begin{tabular}{|c|l|}
\hline Country & Study \\
\hline USA & $\begin{array}{l}\text { Synder et al. [34] Segrin et al. [29], Campbell et al. [24] DEimling et al. [28] Badger et al. [29] Grov et al. [30] Platek et al., Demark-Wahnefried et al. } \\
\text { [36] Kent et al. [32] Synder et al. [13]. Sheriff et al. [37]. Skolarus et al. [39]. Synder et al. [13]. Badger et al. [29]. Miller et al. [25], Miller et al. [25] }\end{array}$ \\
\hline UK & Faithfull et al. [15] Goonewardene SS [21] Goonewardene SS [22] \\
\hline Norway & Grov et al. [30] Takisila et al. [27] Linbohn [27] \\
\hline Netherlands & Mols et al. [16] \\
\hline Finland & Takiskila et al. [27] \\
\hline Australia & Zucca et al. [26], Lynch et al. [35] \\
\hline Sweden & Taylor-Ford et al. [31] \\
\hline Canada & Del Giudice et al. [38] \\
\hline
\end{tabular}

Table 2: Country of origin.

Ashley [20]. Conducted a feasibility study with 886 prostate cancer survivors, over 15 months. Questionnaire based analysis focused on generic, cancer-specific and cancer diagnosis-specific outcome measures. This was conducted by researchers who demonstrated a computer based system, with the potential to provide an affordable UK-scalable technical platform to facilitate and support longitudinal cohort research, and improve understanding of cancer survivors (Moher 'B' quality).

Elliott J, conducted a cross-sectional study with 780 prostate cancer survivors, using a National Health interview survey used to measure outcomes, in terms of health needs. This demonstrated Cancer survivors have ongoing health needs that are not currently being addressed (Moher 'A' quality study).

Harrington [9]. Conducted a cross sectional questionnaire survey on discharge status, provision of time/ information prior to discharge, feelings at discharge and satisfaction with how discharge was managed. They demonstrated discharge of patients from hospital -requires additional time, support and information, again emphasising the extra support needed for this cohort.

Goonewardene [21] 178 Qualitative 1 year Improvement

A quality Goonewardene [21] 178 Quantifiable improvement Qualitative

A quality Goonewardene [22] 500 patients, 20 GPS Cross sectional qualitative study Questionnaire based, GP views on Survivorship programme, to give further support to GPS to manage A quality (Table 2).

The systematic review required narrative analysis. Study designs varied and were conducted by a range of members from the multidisciplinary team including specialist nurses, doctors and in addition, researchers. Number of participants: 258139 patients and 330 primary care physicians.

These papers within this systematic review examine the following topics:

- Cognitive interventions: (Segrin et al.) [23], (Campbell, et al.) [24], (Badger et al.) [23].

- Cancer-related symptoms: (Cherrier), (Van Dis), (Badger et al.) [23], (Miller et al. [25]

- Work (Zucca et al.) [26], (Gilbert et al.) [25].

- Familial impact (Taskilaa et al.) [27]

- Psychological distress (DEimling et al.) [28]. physical and mental health, Depression and anxiety, psychological QOL in PCSs (Badger et al.) [29] Psychometric analysis

- Patient satisfaction (Grov et al.) [30].

- Cognitive and behavioural self-management for symptoms (Faithfull et al.)[15], problem-focused and support-seeking strategies

- Body image (Taylor-Ford et al.) [31].

- Unmet information needs (Kent et al.) [32].

- Physical activity (Livingston et al.) (LaStayo, et al.) [33] (Blanchard et al.) (Synder et al.) [34] (Lynch et al.) [35].

- Lifestyle interventions (Synder, Demark- Wahnefried et al.) [36] (Sheriff et al.) [37]. 
- Ongoing health needs (Elliott et al.) [1] healthcare input (Mols et al.) [16] increased primary health care use (Heins) [16], (Rodgers et al.), (Khan et al.), (SAbatino et al.), (Del Giudice et al.) [38] GP input (Goonewardene et al.) [22].

- Comorbid conditions (Aarts et al.), (Skolarus et al.) [39] (Khan et al.), (Lafata et al.).

- Discharge of patients from hospital (Harrison et al.).

- Survivorship Issues (Baker et al.).

- Fragmented prostate cancer survivorship care and cost (Skolarus et al.) [39].

- Multi-speciality working (Weaver et al.).

- Suboptimal health behaviors (Mosher et al.).

- Patient expectations (Nesse et al.)

- Survivorship care (Goonewardene et al.) [21].

- Impact of cancer (Foley et al.).

- Qol (Mols et al.), (Van Dis et al.), (Blanchard et al.), (Lemasters et al.).

- Patients empowerment (Litwin et al.).

- Return to normal health (Schag et al.).

- Survivorship measures (AVIS et al.).

- Sexual HRQOL, Sexual counselling (Miller et al.) [25].

- Cancer survivorship research (Carmen).

\section{Risk of Bias}

13 studies were of 'C' quality, 27 were of 'A' quality, 36were of ' $B$ ' quality using the criteria of Moher. All studies described withdrawal and dropout rates, including follow-up methodologies, and presented the interventions' outcome results. Blinding was not applicable in any trial. The flow of participants was represented in a consort style diagram in 20 studies. Allocation concealments of participants were not appropriate and the methods used for each study were. Greater than $80 \%$ of participants did provide follow-up data of interest, and outcomes were clearly defined. Only 3 studies had sample size calculated. An adequate summary of results for each outcome was provided, including for non-significant results. Sample results were explicitly defined, as was the method of recruitment and intervention.

\section{Development and Types of Interventions, Components and Delivering of Interventions}

All studies were complex interventions composed of components acting independently and/ or interdependently. Interventions were as follows, below.

- 6 weeks of telephone based cognitive therapy, coping skills training vs. normal care, Campbell, L.C [24].

- 7 weeks of group and individual sessions. Outcomes analysed via questionnaire based on Urinary symptoms were measured before the intervention and again after 4 months of followup through International Prostate Symptom Scores (IPSS), Faithfull [15].

- Telephone counselling and personalised work book, Kent E.E [32].
- Thrice weekly exercise stepping, Outcomes, muscles measurements and mechanical force, Synder [36].

- Physical acivity measured via accelerometer data and waist circumference over 1 week, Lynch [35].

- Telephone analysis: The 7-Day Physical Activity Recall and the Diet History Questionnaire, quality of life; risk for depression; social support; comorbidity; perceived health; self-efficacy for exercising, Demark-Wahnefried [36].

- National Health interview survey used to measure outcomes, Elliott [1].

- Intervention: work book and telephone counselling over 12 month s Assessment over telephone with dietary recall, Synder [36].

- Personalised mail intervention, telephone surveys to assess dietary habits at 1 and 2 years, (Christy et al.).

- Intervention: workbook and unit materials, telephone survey, Demark-Wahenfried [36]

- Point analysis, phone interview, on educational materials and, (Nesse et al.).

- Goonewardene [21].

- Randomised to received tailored vs none tailored diet and exercise intervention. Analysis on Qol via physical activity recall, Ottenbacher.

One study used a telephone delivered quality of life instrument questionnaire [39]. One used focus groups to explore patient experience and benefit of exercise therapy [18]. Mail survey was also used to gain physicians' opinions of routine follow-up. Only study used financial incentives [38]. Study periods varied from cross sectional to three days to analysis over years [39]. All studies had no follow up beyond the specified study period. There were a range of settings used for studies including secondary care, primary care [38] both primary and secondary care university research.

Outcomes measures ranges from sexual and urinary tract functioning to QoL experiences, short and long term preventative outcomes associated with prostate cancer and examination of primary care physicians views on survivorship [38].

Discussion of papers

\section{Impact on Community based Survivorship Care}

This systematic review clearly highlights the large areas of Survivorship Care that are currently being unaddressed (Figure 3).

\section{What patients want}

Survivors report few cancer-related symptoms and high QoL [26]. However some had deteriorated work abilities due to cancer with more problems post therapy [27]. Telephone based interventions are a feasible approach that can successfully enhance coping [24].

\section{Psychological impairment}

Many have psychological distress due to cancer and its treatment. These are strongest predictors of depression and can persist with symptom control [28]. Tailored interventions are appropriate and should be considered in these cases. Further research is however required [29]. 


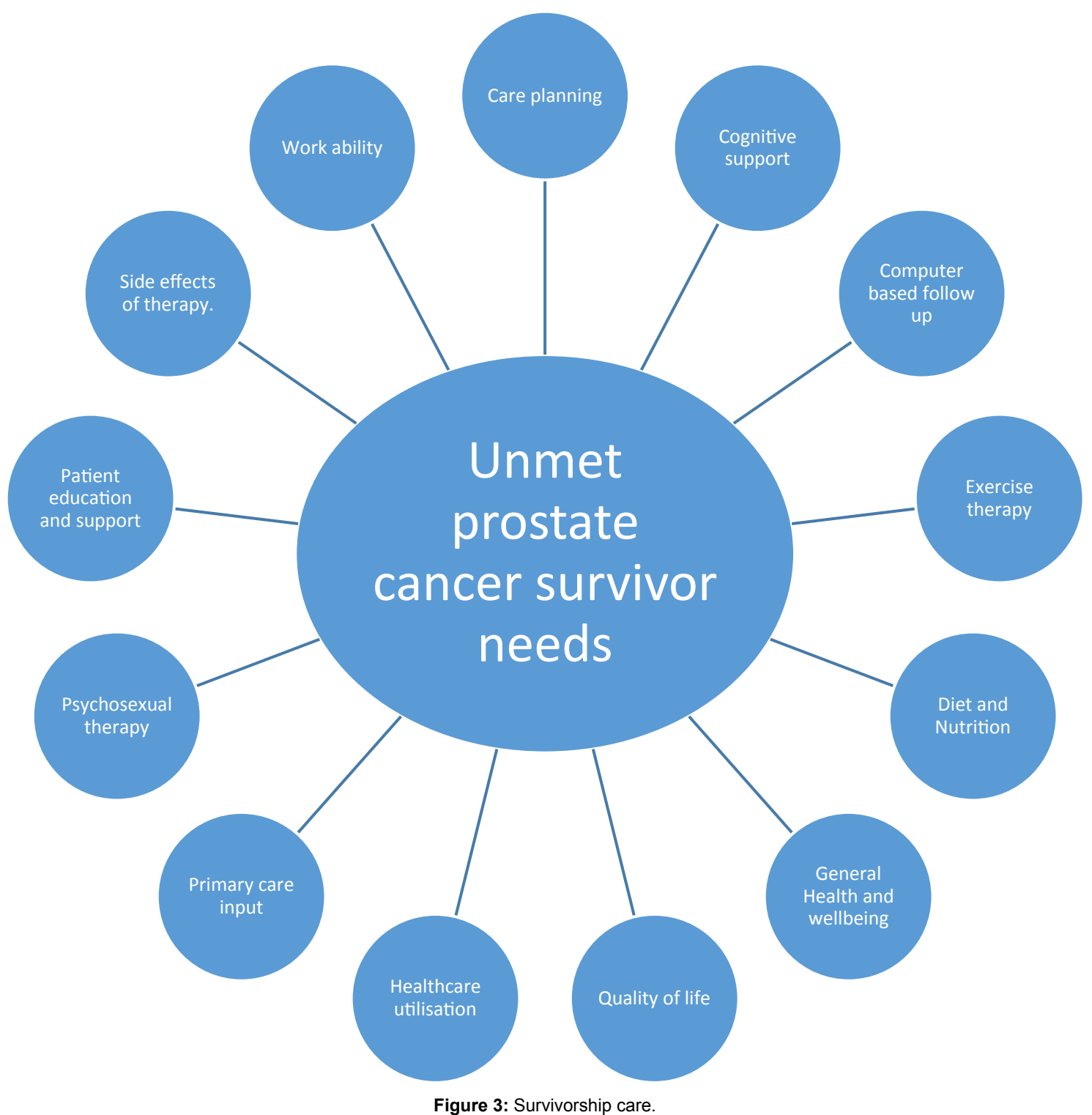

\section{Healthcare utilisation}

Survivors demonstrate a higher rate of decreased self-rated health, more physical impairment and thyroid diseases, daily use of medication and psychotropics and higher level of anxiety [30]. Increased primary health care use is the result, more significant in younger patients. There is a high prevalence of suboptimal health behaviors among older, long-term survivors with appropriate information and support in place, PCPs reported being willing to assume responsibility for the follow-up care of adult cancer survivors [38]. Cardiovascular disease risk factors are common, yet not discussed with relevant healthcare teams. This highlights more inter-speciality working is required. Due to the increased demands, changes in health care, more efficiency, are required to manage increasing demand [16]. The number of physician visits, particularly primary care input, are important factors associated with successful survivorship care [34]. With appropriate information and support in place, multispeciality working together with primary care can manage this cohort.

\section{Cormorbid conditions and side effects of treatment}

Suvivors suffer sexual, urinary, and bowel dysfunction. Another issues which together with erectile dysfunction, could be addressed by adequate Survivorship care. [25].

\section{Social support}

This is increasingly important to the prostate cancer survivor and there carer. As a result any programme which is set up, must also be made accessible to the carer/ relative of the survivors [23]. Direct associations are present between perceived support and the use of problem-focused and support-seeking strategies, whereas inverse associations emerged with self-blame, wishful thinking, and avoidance [40]. Healthcare professionals must aim to promote the former.

\section{Exercise therapy}

Exercise interventions have been shown to improve health related quality of life. There are also clear long term health benefits 
of participating in physical activity programs. Older cancer survivors represent a vulnerable population who we must target and for alteration of health behaviours. This can be a hard-to-reach population [34]. Increasing moderate-to-vigorous activity may assist this population with weight management, however more research is required [35].

\section{Health related Qol}

Most long-term survivors retrospectively report that cancer either positively influenced their lives or had little long-term impact [41]. Those who express Resentment report that pain, physical deformities, and social isolation significantly reduced their long-term HRQL. This is significant, as it highlights a cohort we need to target.

\section{Lifestyle interventions}

Home-based diet and exercise interventions hold promise in improving lifestyle behaviors. However, further research is required [36] even in older survivors [36]. That diet and other lifestyle practices were important predictors of patient QOL [37]. As a cohort, we need to focus on physical, functional and social well-being. [1].

\section{Post treatment care}

Despite high levels of satisfaction, discharge of cancer survivors from hospital follow-up could be improved with the provision of additional time, information and support. Better structuring of the final hospital appointment or a review appointment in primary care at this time could help to ensure that discharge from hospital follow-up is managed optimally for cancer survivors. [29].

\section{Psychosexual}

Only $43 \%$ of men said their partners had encouraged them to find help. This highlights an area of survivorship care which is not properly addressed. This must be corrected to improve sexual rehabilitation in this cohort post therapy.

\section{Cost effectiveness}

None of the studies address cost effectiveness of interventions directly, however one [39] does. This study examined that telephone follow-up is a feasible strategy for or assessing prostate cancer survivor QOL and could provide a low cost, sustainable, and systematic approach to measuring patient-centred outcomes, conducting comparative effectiveness research, and monitoring the quality of prostate cancer care [39].

\section{Statement of main findings}

Our review found a small number of papers of similar research design. All studies reported positive survivorship outcomes or gave further evidence for a way forward. This demonstrates how well adapted the interventions were structured, coupled with the fact that key points were investigated leading to good care. The studies were of moderate quality in relation to the characteristics of their particular design.

Prostate cancer survivors were the focus of these interventions research in all studies. This is a very sizable group, not just in the UK but through the world.

\section{Strengths and limitations}

The search criteria of this review included prostate cancer and survivorship. Interventions of any research design (from a wide range of sources including experts) were assessed and included using the novel survivorship care assessment tool to ensure the inclusion of all relevant interventions previously undertaken in the area. Therefore, this design was robust because previous systematic reviews have limited their search to specific survivorship topics, not looking at survivorship as a holistic package of care. The included studies were assessed for both methodological quality and strength of survivorship care. The review is limited by the different methodological studies. It was a relatively heterogeneous population, indicating the conclusions published are valid. In addition, as only published studies were included, some relevant ongoing studies may have been excluded. The definitions of 'Survivor' have been signposted in this review.

\section{Findings in Relation to Other Survivorship Studies and Trends, Literature}

Concerns regarding permanent physical, psychosocial, and economic effects of cancer treatment were highlighted by the US Institute of Medicine Report [6]. These include impact on life for example, financial, occupational or performance concerns.

The Institute of Medicine produced a report on the focus of survivorship care plans including the chronic effects of cancer, monitoring for and preventing late effects e.g. malignancies, and promoting healthy lifestyles. There is a lack of evidence in this field, with regard to patient follow up and whether it should be led by primary or secondary care, and also a lack of follow up into a patients' wellbeing and quality of life. Survivorship care plans are recommended as an important tool to facilitate communication and allocation of responsibility as part of this. Self -management is part of this, with patient driven assessment of outcomes (National Cancer Intelligence Network) [5].

A survey conducted by Macmillan of 1001 survivors demonstrated current services were falling short of their needs [42]. 94\% said they would expect a full assessment of their on-going needs; $92 \%$ said they would expect to discuss potential side effects of treatment; $89 \%$ would expect a personalised care plan to support them after therapy. There is also a suggestion that $70 \%$ of patients living with and beyond cancer could self-manage their symptoms.

The unmet needs of cancer survivors, the rising numbers, and pressures to utilise resources efficiently [7] are a significant burden on the health system. These issues have been raised by the National Cancer Survivorship Initiative (NCSI) (Giarelli) [8].Which highlighted key shifts in attitude towards care. The focus is now more on recovery and return to work. This includes personalised approach to individual risk assessment and patient self-management.

This is supported by research conducted by the Picker Institute, an independent nonprofit organization dedicated to advancing the principles of patient-centered care. Their research demonstrated $43 \%$ of respondents would have liked more information and advice, $75 \%$ did not hae or did not know if they had, a care plan. $75 \%$ reported not knowing who to contact for advice outside of office hours. Further results from mapping exercises conducted by NCIS include psychological, physical and occupational problems, with a lack of information [7].

The patient consensus meeting concluded patients are not averse to new approaches to follow-up care and support. However they need to have access to good quality information and rapid access to specialist treatment -should they need it.

An important pre-requisite for survivorship care, is a good insight 
into the patients' needs and preferences. Providing cancer patients with information about their disease and treatment helps them to make decisions about treatment. In addition they are able to overcome fear, develop realistic expectations, manage side effects and comply with treatment. Individualised information sessions have been associated with lower anxiety, better psychological well-being and higher treatment satisfaction [43]. Continuity and coordination of care is difficult, with many patients unclear about who is responsible for their ongoing survivorship care [38]. However, to date, there has been little consensus on the value and organisation of follow-up.

One area which is infrequently addressed is that of sexual dysfunction. This affects many prostate cancer survivors. The incidence of varies between $20 \%$ and $88 \%$. Changes in body image, pain, and loss of desire, long-term physical and psychological side effects from cancer treatments can affect sexual functioning.

\section{Other Follow-ups Programmes}

There is a lack of evidence in this field, with regard to patient follow up and whether it should be led by primary or secondary care, and also a lack of follow up into a patients' wellbeing and quality of life. The current method of follow-up involved focusing on cancer as an acute disease, with monitoring for recurrence, and no focus on the physical, social, emotional or psychological impact of being a Cancer Survivor. However, there is some debate as to the efficacy of this [7].

The associated clinical improvement section ran pilot models of improved care and support for survivors [7]. As a result of this, five key phases to survivorship care were identified: care via primary treatment from diagnosis, enable as rapid and full a recovery as possible, ensure recovery is sustained, manage side effects of treatment, monitor for recurrence or disease progression.

This has been taken one step further by the Queensland government, who have developed a framework allowing patients to make and participate in decisions, build and sustain partnerships, possess the capacity to manage the impact of their health in functioning, emotions and interpersonal relationships and monitor and manage symptoms and signs of recurrence.

Interventions based on rehabilitation and self-management requires further research is required.

\section{Conclusion and Recommendations for Future}

This systematic review has defined landmarks for survivorship care: monitoring for recurrence, metastases or side effects and coordination between secondary and primary care. This has also demonstrated a requirement for further holistic support for patients in the survivorship cohort, which is not being addressed. In addition patients with psychological, emotional, social and financial concerns as well as sexual health concerns were also highlighted as not having their problems addressed leading to poorer quality of life. Since writing this systematic review, based on SSGs recommendation, Prostate Cancer Survivorship and Supportive Care has been added as a section to the EAU congress. This is the way forward.

\section{References}

1. Maddams J, Brewster D, Gavin A, Steward J, Elliott J, et al. (2009) 101: 541 547.

2. Improving Outcomes in Cancer (2011) Department of Health Publication.

3. Ganz PA (2009) Survivorship adult cancer survivors.Prim Care 36: 721-41.
4. Centers for Disease Control and Prevention. Cancer survivorship-United States (2004) MMWR Surveill Summ 53: 526-529.

5. National Cancer Intelligence Network UK (2008) Lifetime prevalence for all cancers and colorectal, lung, breast and prostate cancers.

6. Hewitt M, Greenfield W (2006) From Cancer Patient to Cancer Survivor. In Stovall E (eds.) The National Academies Press, Washington.

7. National Cancer Survivorship Initiative (2008)

8. Giarelli E (2004) A model of survivorship in cancer genetic care. Semin Oncol Nurs 20: 196-202.

9. Harrington C, Hansen J, Moskowitz MT, Feuerstein BL, Michael L (2010) It's not over when it's over: Long-term symptoms in cancer survivors: A systematic review. International Journal of Psychiatry in Medicine 40: 163-181.

10. DaviesBedford Hospital, BatehupSupport L, Addenbrooke's Programme (2011) The role of diet and physical activity in breast, colorectal and prostate cancer survivorship. a review of the literature British Journal of Cancer 105: 52-73.

11. Mottet N, Bellmunt J, Bolla M, Joniau S, Mason M, et al. (2011) EAU guidelines on prostate cancer. Part II: Treatment of advanced, relapsing, and castrationresistant prostate cancer. Eur Urol 59: 572-583.

12. Buffart LM, Galvão DA, Brug J, Chinapaw MJM, Newton RU (2014) Evidencebased physical activity guidelines for cancer survivors: Current guidelines knowledge gaps and future research directions Cancer Treatment Reviews 40: 327-340.

13. Snyder DC, Morey Miriam C, Sloane R, Stull V, Cohen HJ, et al. (2009) Reach out to Enhance Wellness in Older Cancer Survivors (RENEW).design, methods and recruitment challenges of a home-based exercise and diet intervention to improve physical function among long-term survivors of breast, prostate, and colorectal cancer. Psycho-Oncology 18: 405-411.

14. Michael E, Galbraith, Regina Fink, Wilkins Gayle G, Couples Surviving Prostate Cancer. Challenges In Their Lives And Relationships Seminars in Oncology Nursing 27: 300-308.

15. Jane Cockle-Hearne, Sara Faithfull (2010) Self-management for men surviving prostate cancer. a review of behavioural and psychosocial interventions to understand what strategies can work,for whom and in what circumstances Psycho-Oncology 19: 909-922.

16. Husson O, Mols F, Van de Poll-Franse LV (2011) The relation between information provision and healthrelated quality of life, anxiety and depression among cancer survivors: a systematic review Annals of Oncology 22: 761-772

17. Barbara Muzzatti , Antonietta Annunziata M (2013) Assessing quality of life in long-term cancer survivors: a review of available tools Supportive care in cancer 21: 3142-3152.

18. Martin E, Bulsara C, Naumann F, Magrani Do Rosario P, Smith C, et al.(2014) What men and women want: The experiences of men and women in a group exercise counselling cancer recovery programme. Journal of Science and Medicine in Sport 14: 117-118.

19. Lyn Thaxton, Emshoff James G, Omar Guessous MA (2005) Prostate Cancer Support Groups.Journal of Psychosocial Oncology 23: 25-40.

20. Jacobs LA, Vaughn DJ, Cotton D, Taichman D, Williams S (2013) In the clinic care of the adult cancer survivor. Annals of Internal Medicine 158: 6-16.

21. Goonewardene SS, Symons M, McCormack G, Makar A (2012) The Worcestershire Prostate Cancer Survivorship Programme:a new concept for long term support and follow-up. Journal of American College of Surgeons 215: 143.

22. Goonewardene SS, Young A, Symons M, Sullivan A, McCormack, et al. (2013) The prostate cancer survivorship program: A new concept for holistic long-term support and follow-up. Journal of Clinical Oncology 30 (5 SUPPL. 1).

23. Chris Segrin, Terry A (2010) Badger Psychological Distress in Different Socia Network Members of Breast and Prostate Cancer Survivors. Researchin Nursing \& Health 33: 450-464

24. Campbell Lisa C, Keefe Francis J, Cindy Scipio BA, McKee Daphne C, et al. (2007) Facilitating Research Participation and Improving Quality of Life for African American Prostate Cancer Survivors and Their Intimate Partners A Pilot Study of Telephone-Based Coping Skills Training CANCER Supplement 109: 2 .

25. Gilbert SM, Miller DC, Hollenbeck BK, Montie JE, Wei JT (2008) Cancer 
survivorship.Challenges and changing paradigms. Journal of Urology 179: 431-438.

26. Zucca Alison C, Allison W (2012) Quality of Life and Physical Symptom Clusters in Long-Term Cancer Survivors Across Cancer Types Journal of Pain and Symptom Management 43: 720-732.

27. Taina Taskilaa, Rami Martikainenb, Pa"ivi Hietanenc, Marja-Liisa Lindbohma (2007) Comparative study of work ability between cancer survivors and their referents. European journal of cancer 43: 914-920.

28. Deimlinga Gary T, Boaz Kahanab, Bowmanc Karen F, Schaefer Michael L (2002) Cancer Survivorship And Psychological Distress In Later Life PsychoOncology 11: 479-494.

29. Badger Terry A, Chris Segrin, Figueredo Aurelio J, Joanne Harrington, Kate Sheppard, et al. (2013) Who benefits from a psychosocial counselling versus educational intervention to improve psychological quality of life in prostate cancer survivors.Psychology \& Health 28: 336-354.

30. Grov Ellen K, Fossål Sophie D, Dahl Alv A Morbidity, life style and psychosocial situation in cancer survivors aged 60-69 years: results from The NordTrøndelag Health Study (The HUNT-II Study)

31. Megan Taylor-Ford, Meyerowitz Beth E, D'Orazio Lina M, Christie Kysa M, et al. (2013) Body image predicts quality of life in men with prostate cancer. Psycho-Oncology 22: 756-761.

32. Kent Erin E, Neeraj K, Arora C, Julia H, Rowland B, et al. (2012) Health information needs and health-related quality of life in a diverse population of long-term cancer survivors. Patient Education and Counseling 89: 345-352.

33. LaStayo Paul C, Marcus V, Dibble Lee E, Smith Sheldon B, Beck Susan L, et al. (2009) Psycho-Oncology 18: 429-439.

34. Snyder CF, Frick KD, Herbert RJ, Blackford AL, Neville BA, et al. (2011) Preventive care in prostate cancer patients: following diagnosis and for fiveyear survivors. Journal of Cancer Survivorship 5: 283-91.
35. Lynch BM, Dunstan DW, Winkler E, Healy GN, Eakin E, et al. (2011) Objectively assessed physical activity, sedentary time and waist circumference among prostate cancer survivors: findings from the National Health and Nutrition Examination Survey. European Journal of Cancer Care 20: 514-519.

36. Demark-Wahnefried W, Morey Miriam C, Clipp Elizabeth C, Pieper Carl F Snyder DC, et al. (2003) Leading the Way in Exercise and Diet: intervening to improve function among older breast and prostate cancer survivors. Controlled Clinical Trials 24: 206-223.

37. Sheriff Sara K, Shohara Ryo A, Dumican Sarah B, Small Eric J, Carroll Peter $\mathrm{R}$, et al. (2005) Lifestyle Correlates of Health Perception and Treatment Satisfaction in a Clinical Cohort of Men with Prostate Cancer. Clinical Prostate Cancer 3: 239-245.

38. Del Giudice ME, Grunfield E, Harvey B J, Piliotis E, Verma S (2009) Primary Care Physicians views of routine follow-up of cancer survivors 20: 3338-3345.

39. Skolarus TA, Holmes-Rovner M, Hawley ST, Dunn RL, Barr KL, et al. (2012) Monitoring quality of life among prostate cancer survivors: The feasibility of automated telephone assessment. Urology 80: 1021-1026.

40. Ptacek JT, Pierce Gregory R, Ptacek John J (2002) The Social Context of Coping with Prostate Cancer, Journal of Psychosocial Oncology 20: 61-80.

41. Saad F, Finelli A, Dranitsaris G, Goldenberg L, Bagnell S (2006) Canadian surgical wait times (SWAT) initiative. Can J Urol 13: 16-24.

42. Productivity and Prevention National Cancer Institute (2012) The Quality, Innovation NCCS Charter.

43. Hummel S, Simpson EL, Hemingway P, Stevenson MD, Rees A (2010) Intensity-modulated radiotherapy for the treatment of prostate cancer: a systematic review and economic evaluation. Health Technol Assess 14: 1-108. 